\title{
Inflammatory Indicator and Hematological Indices in Contrast Induced Nephropathy among Patients Receiving Coronary Intervention: A Systematic Review and Meta-Analysis
}

Xiaoyan $\mathrm{Wu}^{1} \mathrm{MD}$, Chao $\mathrm{Ma}^{2} \mathrm{MD}$, Daqing $\mathrm{Sun}^{1} \mathrm{MD}$, Guojing Zhang ${ }^{1} \mathrm{MD}$, Jinmiao Wang $^{1}$ MD, Enyuan Zhang ${ }^{3 *}$ MD

1. Department of Pediatric Surgery, Tianjin General Hospital, Tianjin, China

2. Department of General Surgery, Tianjin General Hospital, Tianjin, China

3. Department of Cardiology, Tianjin Chest Hospital, Tianjin, China

*Correspondence author: Enyuan Zhang Email: doctorenyuan@126.com 


\begin{abstract}
Background: Strong indicators of inflammation, such as C-reactive protein (CRP), hypersensitive CRP (hs-CRP), and a series of hematological indices, including platelet to lymphocyte ratio (PLR), neutrophil to lymphocyte ratio (NLR), hematocrit (HCT) and red blood cell distribution width (RDW), are regarded related with the incidence of contrast induced nephropathy (CIN) closely. Whereas, it remains unclear whether they can function as predictors of CIN onset. The objective of this meta-analysis was to determine the relationship between above indicators and CIN incidence among patients receiving coronary intervention.
\end{abstract}

Methods: Clinical studies were retrieved from the electronic databases of PubMed, EMBASE, Google Scholar, Clinical Trials, and science direct from their inception to June 3rd, 2020. Meta-analysis was performed on pool eligible studies. Two reviewers screened all titles and abstracts and independently assessed all articles.

Results: A total of 26 studies involving 29,454 patients were included in the metaanalysis. Pooled analysis results revealed that patients with higher CRP (odds ratio $[\mathrm{OR}]=1.06,95 \%$ confidence interval $[\mathrm{CI}]: 1.01-1.12, \mathrm{P}=0.02)$, hs-CRP $(\mathrm{OR}=1.03,95 \%$ CI: 1.01-1.06, $\mathrm{P}=0.004), \mathrm{NLR}(\mathrm{OR}=1.11,95 \% \mathrm{CI}: 1.01-1.20, \mathrm{P}=0.02), \mathrm{RDW}$ $(\mathrm{OR}=1.35,95 \%$ CI: $1.19-1.53, \mathrm{P}<0.00001)$, and lower HCT $(\mathrm{OR}=0.94,95 \%$ CI: $0.92-$ 0.97, $\mathrm{P}=0.0003$ ) all exhibited significantly higher $\mathrm{CIN}$ rates, but there was no significant association between PLR and CIN risk (OR=1.12, 95\% CI: 0.99-1.26, $\mathrm{P}=0.07)$

Conclusion: The meta-analysis reported here demonstrates that pre-angiography 
CRP/hs-CRP and some hematological indices are associated with CIN.

Keywords: C-reactive protein; platelet to lymphocyte ratio; neutrophil to lymphocyte ratio; hematocrit; red blood cell distribution width; contrast induced nephropathy; coronary intervention

Abbreviations: $\mathrm{CIN}=$ Contrast-induced nephropathy; $\mathrm{AKI}=$ acute kidney injury; $\mathrm{CRP}$ $=\mathrm{C}$-reactive protein; hs-CRP $=$ hypersensitive $\mathrm{CRP} ; \mathrm{PLR}=$ platelet to lymphocyte ratio; $\mathrm{NLR}=$ neutrophil to lymphocyte ratio; RDW = red blood cell distribution width; HCT $=$ hematocrit; MACEs $=$ major adverse cardiac events; NOS = Newcastle-Ottawa Quality Score; ORs $=$ odds ratios; $\mathrm{CI}=$ confidence interval; $\mathrm{SAP}=$ stable angina pectoris; NSTE-ACS = non-ST-segment elevation acute coronary syndromes; STEMI $=$ ST-segment elevation myocardial infarction; $\mathrm{PCI}=$ percutaneous transluminal coronary intervention; PTCA = Percutaneous Transluminal Coronary Angioplasty; DM $=$ diabetes mellitus; $\mathrm{HTN}=$ hypertension; $\mathrm{Hb}=$ hemoglobin; $\mathrm{LVEF}=$ left ventricular ejection fraction; eGFR = estimated glomerular filtration rate; MVD = multi-vessel disease; $\mathrm{CV}=$ contrast volume

1. Introduction

Contrast-induced nephropathy $(\mathrm{CIN})$ is an increasingly common cause of iatrogenic acute kidney injury (AKI), leading to the extension of hospitalization, 
increasement of the short- and long-term mortality, and accelerated progression of underlying chronic kidney disease.(1) Inflammation contribute to the pathogenesis of CIN in the setting of acute coronary syndrome(2) and diabetic kidney disease.(3) A study recruiting 423 patients highlights the importance of inflammation as a risk factor in the development of CIN.(4) Besides, inhibiting inflammation could attenuate CIN, simultaneous with anti-oxidative stress, anti-apoptosis, anti-autophagy and improve renal blood perfusion.(5-7)

C-reactive protein (CRP) and hypersensitive CRP (hs-CRP) are strong indicators of inflammation and related with prognosis in patients undergoing coronary intervention. A meta-analysis based on 33 studies involving 34,367 patients suggested that pre-procedural serum CRP level is a valuable predictor of major adverse cardiac events (MACEs), all-cause death, myocardial infarction, coronary revascularization, and clinical restenosis in patients undergoing percutaneous coronary intervention (PCI).(8)

Recently, several easily calculated hematological indices have been found to show prognostic value in patients receiving coronary intervention. High pre-intervention platelet to lymphocyte ratio (PLR) and neutrophil to lymphocyte ratio (NLR) are independent predictors of long-term adverse clinical outcomes in patients with unstable angina and non-ST elevated myocardial infarction receiving successful PCI.(9) They could also predict the no-reflow phenomenon and proceed accurate risk stratification in patients with ST elevated myocardial infarction undergoing primary PCI.(10) A metaanalysis enrolling 14 studies of 10,245 patients shows that NLR is a predictor of 
hospitalization and long-term prognosis in patients with ST elevated myocardial infarction after PCI.(11) Besides judging whether anemia exists, hematocrit (HCT) at baseline and the drop after PCI should be recognized as important risk factors for adverse outcomes after PCI.(12) Red blood cell distribution width (RDW) combined with GRACE score could independently predict long-term MACEs in STsegment elevation myocardial infarction (STEMI) patients undergoing primary PCI.(13) Significant association between in-hospital and 6-month mortality as well as the occurrence of MACEs and high RDW was also found.(14)

Whereas, whether above inflammatory indicators and hematological indices before coronary intervention can function as predictors of CIN onset remains ambiguous. Therefore, the primary purpose of our study was to evaluate the predictive value of them for CIN using meta-analytical methodology.

2. Materials and methods

\subsection{Literature search strategy}

To identify studies involving the association between inflammatory indicators (CRP/hs-CRP), a serious of hematological indices (PLR, NLR, HCT, RDW) and CIN incidence following coronary intervention, a literature search was conducted among 5 English databases (PubMed, EMBASE, Google Scholar, Clinical Trials, and science direct), from their inception to July 31, 2018. We checked these electronic databases using the following searching strategies: ("C-reactive protein" or "high sensitivity Creactive protein" or "CRP" or "hs-CRP" or "neutrophil to lymphocyte ratio" or 
"neutrophil/lymphocyte" or "NLR" or "platelet to lymphocyte ratio" or "platelet/lymphocyte" or "PLR" or "red blood cell distribution width" or "RDW" or "hematocrit" or "HCT") and (“contrast induced nephropathy" or "acute kidney injury"). Additionally, we performed a computerized search of abstracts. Finally, we screened the references in all relevant articles to identify additional articles that were not retrieved during the initial literature search.

\subsection{Selection criteria}

Our meta-analysis included all studies meeting the following criteria: the definition of CIN: serum creatinine change $\geq 25 \%$ or $\geq 44.2 \mathrm{mmol} / 1(0.5 \mathrm{mg} / \mathrm{dL})$ within short time following coronary angiography and PCI if necessary, patients receiving carotid, peripheral artery angiography or transcatheter aortic valve implantation were excluded; all patients examined at least one of the above inflammatory indicators or hematological indices before coronary intervention; results were part of an original analysis and provided with odds ratio (ORs) and 95\% confidence interval (CI) to present the risk of CIN; papers were published in English. We only selected the articles published in peer-reviewed journals and excluded reviews, letters, and meeting abstracts.

\subsection{Quality assessment}

Through independently screening of titles and abstracts by 2 reviewers, the initial relevance evaluation was implemented. The full text was obtained if either reviewer considered any titles or abstracts met the eligibility criteria. The quality and bias risk of the selected papers were critically appraised separately by 2 reviewers. Quality 
assessment was conducted for each of the eligible studies by using the validated Newcastle-Ottawa Quality Assessment Scale (NOS) to reflect the combined scores of selection, comparability and outcome description.(15) This scale is composed of 8 items that assess patient selection, study comparability, and outcome with scores ranging from 0 to 9 . In our meta-analysis, studies with a score $>6$ were graded as high quality.(16) Eventual consensus governance resolved disagreements.

\subsection{Data extraction}

Information from each study was abstracted independently by 2 investigators using a standardized data extraction form, predesigned on the basis of the Cochrane Consumers and Communication Review Group data extraction template. Any disagreement over extracted data was resolved through discussion until the 2 investigators reached a consensus opinion. The primary endpoint was CIN onset. The following information was recorded for each publication: first author's name, publication year, country of origin, cohort design, patient status, numbers of patients, CIN definition, NOS score, detailed patient information including sex distribution, age, rate of hypertension, diabetes mellitus, multi-vessel disease, CIN, value of hemoglobin, left ventricular ejection fractions, estimated glomerular filtration rate, inflammatory indicators or hematological indices in individual studies. When key pieces of information were not present in articles, the corresponding author was contacted. The missing information was classified as "not applicable" when the whole dataset could not be obtained.

\subsection{Statistical methods}


Dichotomous results were summarized as pooled ORs and 95\% CIs around the point estimates. ORs was abstracted or calculated to quantitatively evaluate the association between value of individual inflammatory indicators, hematological indices and the CIN incidence rate. The overall pooled effect was assessed using the z-statistic with a $p$-value $<0.05$ representing statistical significance. Heterogeneity between the

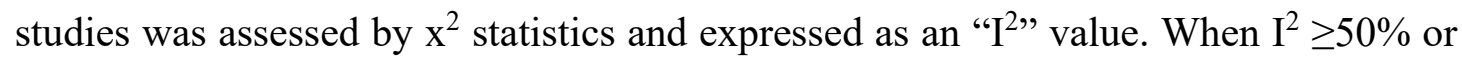
the P-value for the $\mathrm{I}^{2}$ statistic was $<0.05$, which indicated significant heterogeneity across the studies, the pooled estimate was calculated using a random effects model and if the data were contrary, a fixed effect model was adopted. All statistical analyses were carried out using RevMan 5.3 software. All analyses were based on previous published researches, thus no ethical approval or patient consent was required.

\section{Results}

\subsection{Search results}

The search strategy yielded 1689 potentially relevant references in the electronic databases. We initially excluded 858 duplicated publications. Upon review of the remaining abstracts, we further removed 747 more articles for reasons of ineligibility. According to the inclusion criteria established for the present study, an additional 58 articles were excluded. We thus finally selected 26 studies(17-42), which consisted of a cohort of 29,454 patients receiving coronary intervention (figure 1).

All of the 26 selected studies assessed the association analysis between preangiography CRP/hs-CRP, hematological indices and CIN, 13 of them contained the relationship between $\mathrm{CIN}$ and CRP/hs-CRP(19, 23, 24, 27, 28, 32, 34-36, 38, 39, 41, 
43), while 5 of them reported $\operatorname{NLR}(25,36,38,39,42), 4$ explored PLR(19, 26, 36, 42), $5 \operatorname{RDW}(29,30,40,41,44)$ and $7 \operatorname{HCT}(17,18,21,31,33,37,40)$. The definition of CIN was almost consistent among the enrolled studies: serum creatinine change $\geq 25 \%$ or $\geq 44.2 \mathrm{mmol} / 1(0.5 \mathrm{mg} / \mathrm{dL})$, only 3 studies $(22,25,39)$ preserve one of them. The vast majority of patients received coronary intervention unless not serious enough or coronary artery bypass grafting is needed. Most studies focus on patients with acute coronary syndrome, except for $3(17,22,31)$ on patients receiving elective coronary angiography and intervention if necessary, one(18) on patients without STEMI and one(41) on patients with stable angina pectoris. Most of our included studies are from China and Turkey except for 3 from Japan(24, 30, 31), 2 from America(18, 44), 2 from Korea(17, 27), 1 from Iran(21), 1 from Poland(40) and 1 from Italy(22).

A summary of the available information included in the present meta-analysis is provided in Table 1 . NOS score was $>6$ in each selected study. Basic information and situation, medical history, severity of vascular disease, contrast volume and individual pre-angiographic value of inflammatory indicators or hematological indices were shown in Table 2.

\subsection{Statistical pooling}

The mean LVEF variated extremely among individual studies, range from $43.5 \%(40)$ to $65.6 \%$,(41) while basic level of eGFR range from $63.6 \mathrm{ml} / \mathrm{min}(24)$ to $103.8 \mathrm{ml} / \mathrm{min}(42)$, the average contrast volume distributed widely from $122 \mathrm{ml}(34)$ to $271 \mathrm{ml}(33,37)$, the overall $\mathrm{CIN}$ incidence rate occurred highest in study from Nakamura(31) (up to 19.7\%), patients in research from $\operatorname{Sun}(36)$ suffered the lowest CIN incidence rate of $4.4 \%$, with the highest level of hemoglobin $(15.5 \mathrm{~g} / \mathrm{L})$ and fourth 
highest level of eGFR $(93.8 \mathrm{ml} / \mathrm{min})$. The pooled analysis results revealed that patients with higher $\mathrm{CRP}(\mathrm{OR}=1.06,95 \% \mathrm{CI}: 1.01-1.12, \mathrm{P}=0.02$, figure $2 \mathrm{a})$, hs-CRP $(\mathrm{OR}=1.03$, 95\% CI: $1.01-1.06, \mathrm{P}=0.004$, figure $2 \mathrm{~b}), \mathrm{NLR}(\mathrm{OR}=1.11,95 \% \mathrm{CI}: 1.01-1.20, \mathrm{P}=0.02$, figure 2c), $\mathrm{RDW}(\mathrm{OR}=1.35,95 \% \mathrm{CI}: 1.19-1.53, \mathrm{P}<0.00001$, figure $2 \mathrm{~d}$ ), and lower HCT $(\mathrm{OR}=0.94,95 \% \mathrm{CI}: 0.92-0.97, \mathrm{P}=0.0003$, figure $2 \mathrm{e})$ all exhibited significantly higher CIN rates, but there was no significant association between PLR and CIN risk $(\mathrm{OR}=1.12$, 95\% CI: $0.99-1.26, \mathrm{P}=0.07$, figure $2 \mathrm{f})$. No significant heterogeneity was found among RDW subgroup, so we performed a sensitivity analysis among other subgroups by recalculating ORs and $\mathrm{I}^{2}$ with 1 study removed and all others included from the pooled estimate, we assessed the influence of each study on the overall estimate. Sensitivity analysis showed no substantial difference in $\mathrm{I}^{2}$ and pooled ORs when any single study was excluded in most subgroups, which indicated that the conclusion was robust. When we performed a sensitivity analysis in HCT subgroup, we found a substantial difference in $\mathrm{I}^{2}$ without change in pooled OR when the study of Nakamura(31) was excluded. The heterogeneity may come from the relatively smaller sample $(n=66)$, higher level of eGFR and higher incidence of CIN due to the wide range of definition time (2-5 days after procedure).

Then we performed subgroup analyses by design, study race (Xanthoderm vs. Caucasian), and whether ACS or non-ACS (Table 3). The country and publication year distribution of our included studies were showed in the figure 3.

\subsubsection{Publication bias.}

In the meta-analysis, funnel plots were generally asymmetrical. These results indicated that publication bias was significant across the included studies.

\section{Discussion}

To our knowledge, so far this is the first systemic review and meta-analysis to investigate the impact of several preprocedural inflammatory indicators and hematological indices on the occurrence of CIN in patients who received coronary intervention. The results from our study suggested that there was a significant link 
between increased preprocedural CRP levels and the incidence of CIN. High-risk patients can often be identified ahead of time to prevent potential CIN as far as possible.

As symbol of inflammation, CRP is significantly associated with the risk of CIN, mainly for the purpose that systemic inflammation increases the kidneys' vulnerability to the local inflammatory processes that are elicited by contrast medium reabsorption.(45) Elevated CRP levels are also associated with endothelial injury and impaired vasodilation, which may lead to acute renal damage and progressive loss of kidney function.(46) As categorical variable, hs-CRP also verify its strong prognostic value of CIN incidence. Individual studies respectively pointed out hs-CRP $>6.50 \mathrm{mg} / \mathrm{L}$, hs-CRP >3mg/L and hs-CRP $>7.3 \mathrm{mg} / \mathrm{L}$ were significantly associated with the occurrence of CIN in patients with STEMI(47) and acute coronary syndrome(48) undergoing PCI and in patients undergoing coronary angiography(49) after adjusting for potential confounders. Although not meeting our inclusion criteria due to statistical reason, log hs-CRP still proved to be independent predictors of CIN in multivariable logistic regression analysis.(50-52) An analysis based on PRATO-ACS data showed the magnitude of CIN rescue attributable to rosuvastatin pre-treatment was substantially greater in patients with higher baseline hs-CRP than in patients with lower levels.(53) besides PCI, Preoperative CRP level is also a predictor of postoperative AKI in patients undergoing coronary artery bypass grafting.(54) Elevated hsCRP was associated with subsequent risk of AKI and chronic kidney disease progression in post-myocardial infarction patients, irrespective of baseline renal function(55).

A serious of studies have focused on the mechanism of CRP level and CIN development, CRP was proved to promote AKI by impairing G1/S-dependent tubular epithelium cell regeneration(56) and via Smad3-dependent inhibition of CDK2/cyclin $\mathrm{E}(57)$, which suggest that targeting CRP signalling may offer a new therapeutic potential for AKI. Animal experiment offer the result that downregulation of autophagy is associated with severe ischemia-reperfusion-induced AKI in overexpressing CRP mice, which suggested CRP render the kidney more susceptible to ischemic/oxidative injury by down-regulating autophagy flux.(58) CRP velocity might be an independent and rapidly measurable biomarker for AKI following primary PCI 
in STEMI patients(59).

Although we failed to get the accurate association between PLR and CIN incidence, a large-scale supported the potential possibility, they demonstrated that being in the PLR 4th quartile was significantly associated with an increased risk of developing CIN (OR 2.26, 95\%CI 1.25-4.09, p<0.007), which was not included in our meta-analysis because the overall OR value was not available.(60) PLR >177.5 was found an independent predictive factor for CI-AKI.(61) Turkmen et al.(62) found higher PLR always meant higher levels of inflammation among patients with end-stage renal disease. Besides, on-admission PLR levels in CIN group are signifificantly higher than those of non-CIN group among patients with acute coronary syndrome(63).

The NLR provides a simple but promising evaluation for systemic inflammation and it is used widely as a prominent marker for cardiovascular diseases(64). While considering the CIN risk purely, NLR still proved to be a reliable inflammatory prognostic marker, no matter among patients with $\operatorname{STEMI}(25,36)$ non-ST-segment elevation acute coronary syndrome(65) or peripheral artery disease(66). The progression of renal dysfunction in heart failure patients with reduced left ventricular ejection fraction was associated with NLR level(67). Besides, NLR predicted the worsening of the renal function in diabetic patients(68).

More than one decade ago, HCT level has been enrolled into the Mehran score to evaluate the risk of CIN occurrence, due to its relationship with anemia(69). Subsequently, a study enrolling 6,773 consecutive patients from Nikolsky and Mehran(70) had identified lower baseline HCT as an independent predictor of CIN no matter whether chronic kidney disease exist, a significant increase of CIN risk was found along with each $3 \%$ decrease in baseline hematocrit, the additional association between HCT and inflammation maybe responsible for the former result and our metaanalysis. The Cholesterol and Recurrent Events study reported that higher values of RDW may reflect an underlying inflammatory state(71), which play a crucial role in the pathogenesis of CIN. Besides, abnormal RDW has also been found to be associated with pre-existing impaired renal function, in patients with acute myocardial infarction(72), under hemodialysis state(73), and even kidney transplant recipients,(74) 
independent of comorbidity, iron deficiency, inflammation, and nutritional status. Multivariable Analyses showed RDW (per additional 1\%) could independently predict postoperative AKI in a validation cohort of 333 pediatric patients(75) and a larger matched cohort patients from another study including 3146 patients undergoing cardiac $\operatorname{surgery}(76)$.

The strength of this study is that it is the first meta-analysis that consolidates the available information regarding the performance of some inflammatory indicators and hematological indices in predicting CIN. There are several limitations to the present meta-analysis. First, our analysis was based mainly on findings from observational studies, which might contain a higher number of confounding factors than randomized controlled clinical trials. Second, our analysis only contained published studies. Potential publication bias represents a concern, since positive results are more likely to be reported than negative observations. Therefore, more detailed prospective data is needed for future analyses to determine efficient combination of above indicators for further improving the predictive efficiency in clinical practice.

\section{Conclusion}

In conclusion, our findings support the hypothesis that a serious of easily acquired inflammatory indicators (CRP, hs-CRP) and hematological indices (NLR, HCT, RDW) are associated with the increasing incidence of CIN, which could help develop algorithms to identify patients at increased risk for CIN who would then be subjected to increased preventive measures. Large-scale prospective studies will be required before we add some of them into novel risk scores. 
a. competing interests

There are no competing interests in our study.

b. funding

The authors received no financial support for the research, authorship, and/or publication of this article.

c. data sharing statement

No additional unpublished data are available

Figure legends

Figure 1. Flow diagram of the literature search and study selection process

Figure 2a. Summary of the odds ratio of the association between the risk of contrast induced nephropathy and C-reactive protein

Figure $2 \mathrm{~b}$. Summary of the odds ratio of the association between the risk of contrast induced nephropathy and hypersensitive C-reactive protein

Figure 2c. Summary of the odds ratio of the association between the risk of contrast induced nephropathy and neutrophil to lymphocyte ratio

Figure $2 \mathrm{~d}$. Summary of the odds ratio of the association between the risk of contrast induced nephropathy and red blood cell distribution width

Figure 2e. Summary of the odds ratio of the association between the risk of contrast induced nephropathy and hematocrit

Figure $2 \mathrm{f}$. Summary of the odds ratio of the association between the risk of contrast induced nephropathy and platelet to lymphocyte ratio 
Figure 3a Distribution of studies according to the country.

Figure $3 b$ Distribution of studies according to publication year.

\section{References:}

1. McCullough PA and Sandberg KR: Epidemiology of contrast-induced nephropathy. REV CARDIOVASC MED 4 Suppl 5: S3-S9, 2003

2. Ulus T, Isgandarov K and Yilmaz AS, et al.: Monocyte to High-Density Lipoprotein Ratio Predicts Contrast-Induced Nephropathy in Patients With Acute Coronary Syndrome. ANGIOLOGY 69: 909-916, 2018

3. Onk D, Onk OA and Turkmen K, et al.: Melatonin Attenuates Contrast-Induced Nephropathy in Diabetic Rats: The Role of Interleukin-33 and Oxidative Stress. Mediators Inflamm 2016: 9050828, 2016

4. Kwasa EA, Vinayak S and Armstrong R: The role of inflammation in contrast-induced nephropathy. Br J Radiol 87: 20130738, 2014

5. Zhao Q, Yin J and Lu Z, et al.: Sulodexide Protects Contrast-Induced Nephropathy in SpragueDawley Rats. CELL PHYSIOL BIOCHEM 40: 621-632, 2016

6. Lu Z, Cheng D and Yin J, et al.: Antithrombin III Protects Against Contrast-Induced Nephropathy. EBIOMEDICINE 17: 101-107, 2017

7. Zhao B, Zhao Q, Li J, Xing T, Wang F and Wang N: Renalase protects against contrast-induced nephropathy in Sprague-Dawley rats. PLOS ONE 10: e116583, 2015

8. Bibek SB, Xie Y, Gao JJ, Wang Z, Wang JF and Geng DF: Role of pre-procedural C-reactive protein level in the prediction of major adverse cardiac events in patients undergoing percutaneous coronary intervention: a meta-analysisof longitudinal studies. INFLAMMATION 38: 159-169, 2015

9. Cho KI, Ann SH, Singh GB, Her AY and Shin ES: Combined Usefulness of the Platelet-toLymphocyte Ratio and the Neutrophil-to-Lymphocyte Ratio in Predicting the Long-Term Adverse Events in Patients Who Have Undergone Percutaneous Coronary Intervention with a Drug-Eluting Stent. PLOS ONE 10: e133934, 2015

10. Vakili H, Shirazi M, Charkhkar M, Khaheshi I, Memaryan M and Naderian M: Correlation of platelet-to-lymphocyte ratio and neutrophil-to-lymphocyte ratio with thrombolysis in myocardial infarction frame count in ST-segment elevation myocardial infarction. EUR J CLIN INVEST 47: 322327, 2017

11. Zhang S, Diao J and Qi C, et al.: Predictive value of neutrophil to lymphocyte ratio in patients with acute ST segment elevation myocardial infarction after percutaneous coronary intervention: a metaanalysis. BMC Cardiovasc Disord 18: 75, 2018

12. Maluenda $\mathrm{G}$, Lemesle $\mathrm{G}$ and Collins $\mathrm{SD}$, et al.: The clinical significance of hematocrit values before and after percutaneous coronary intervention. AM HEART J 158: 1024-1030, 2009

13. Chang XW, Zhang SY and Wang H, et al.: Combined value of red blood cell distribution width and global registry of acute coronary events risk score on predicting long-term major adverse cardiac events in STEMI patients undergoing primary PCI. Oncotarget 9: 13971-13980, 2018

14. Bozorgi A, Khaki S and Mortazavi SH, et al.: Effect of Baseline Red Blood Cell Distribution Width on Short- and Intermediate-term Mortality of Patients Under Primary Percutaneous Coronary 
Intervention: A Survival Analysis. Crit Pathw Cardiol 15: 69-74, 2016

15. Cota GF, de Sousa MR, Fereguetti TO and Rabello A: Efficacy of anti-leishmania therapy in visceral leishmaniasis among HIV infected patients: a systematic review with indirect comparison. PLoS Negl Trop Dis 7: e2195, 2013

16. Fu X, Zhou Q and Zhang X: Efficacy Comparison Between Total Laryngectomy and Nonsurgical Organ-Preservation Modalities in Treatment of Advanced Stage Laryngeal Cancer: A Meta-Analysis. Medicine (Baltimore) 95: e3142, 2016

17. Cho JYM, Jeong MHMP and Hwan Park SM, et al.: Effect of contrast-induced nephropathy on cardiac outcomes after use of nonionic isosmolar contrast media during coronary procedure. J CARDIOL 56: 300-306, 2010

18. Dangas G, Iakovou I and Nikolsky E, et al.: Contrast-induced nephropathy after percutaneous coronary interventions in relation to chronic kidney disease and hemodynamic variables. AM J CARDIOL 95: 13-19, 2005

19. Demircelik MB, Kurtul A, Ocek H, Cakmak M, Ureyen C and Eryonucu B: Association between Platelet-to-Lymphocyte Ratio and Contrast-Induced Nephropathy in Patients Undergoing Percutaneous Coronary Intervention for Acute Coronary Syndrome. CARDIORENAL MED 5: 96-104, 2015

20. Elhosseiny S, Akel T, Mroue J, Tathineni P, El Sayegh S and Lafferty J: The Value of Adding Red Cell Distribution Width to Mehran Risk Score to Predict Contrast-induced Acute Kidney Injury in Patients with Acute Coronary Syndrome. Cureus

21. Eshraghi A, Naranji-Sani R and Pourzand H, et al.: Pentoxifylline and prevention of contrastinduced nephropathy: Is it efficient in patients with myocardial infarction undergoing coronary angioplasty? ARYA Atheroscler 12: 238-242, 2016

22. Evola S, Lunetta $\mathrm{M}$ and Macaione F, et al.: Risk factors for contrast induced nephropathy: A study among Italian patients. Indian Heart Journal 64: 484-491, 2012

23. Gao F, Zhou YJ, Zhu X, Wang ZJ, Yang SW and Shen H: C-reactive protein and the risk of contrastinduced acute kidney injury in patients undergoing percutaneous coronary intervention. AM J NEPHROL 34: 203-210, 2011

24. Gohbara M, Hayakawa A and Akazawa Y, et al.: Association Between Acidosis Soon After Reperfusion and Contrast-Induced Nephropathy in Patients With a First-Time ST-Segment Elevation Myocardial Infarction. J AM HEART ASSOC 6, 2017

25. Kaya A, Kaya Y and Topcu S, et al.: Neutrophil-to-lymphocyte ratio predicts contrast-induced nephropathy in patients undergoing primary percutaneous coronary intervention. ANGIOLOGY 65: 5156,2014

26. Kocas C, Yildiz A and Abaci O, et al.: Platelet-to-Lymphocyte Ratio Predicts Contrast-Induced Nephropathy in Patients With Non-ST-Segment Elevation Acute Coronary Syndrome. ANGIOLOGY 66: 964-968, 2015

27. Koo HM, Doh FM and Ko KI, et al.: Diastolic dysfunction is associated with an increased risk of contrast-induced nephropathy: a retrospective cohort study. BMC NEPHROL 14: 146, 2013

28. Kurtul A, Yarlioglues M and Duran M: Predictive Value of CHA2DS2-VASC Score for ContrastInduced Nephropathy After Percutaneous Coronary Intervention for Acute Coronary Syndrome. AM J CARDIOL 119: 819-825, 2017

29. Kurtul A, Yarlioglues M and Murat SN, et al.: Red cell distribution width predicts contrast-induced nephropathy in patients undergoing percutaneous coronary intervention for acute coronary syndrome.

ANGIOLOGY 66: 433-440, 2015 
30. Mizuno A, Ohde S, Nishizaki Y, Komatsu Y and Niwa K: Additional value of the red blood cell distribution width to the Mehran risk score for predicting contrast-induced acute kidney injury in patients with ST-elevation acute myocardial infarction. J CARDIOL 66: 41-45, 2015

31. Nakamura T, Sugaya T, Node K, Ueda Y and Koide H: Urinary Excretion of Liver-Type Fatty AcidBinding Protein in Contrast Medium-Induced Nephropathy. AM J KIDNEY DIS 47: 439-444, 2006

32. Oksuz F, Yarlioglues M and Cay S, et al.: Predictive Value of Gamma-Glutamyl Transferase Levels for Contrast-Induced Nephropathy in Patients With ST-Segment Elevation Myocardial Infarction Who Underwent Primary Percutaneous Coronary Intervention. AM J CARDIOL 116: 711-716, 2015

33. Rencuzogullari I, Cagdas M and Karakoyun S, et al.: Association of Syntax Score II with Contrastinduced Nephropathy and Hemodialysis Requirement in Patients with ST Segment Elevation Myocardial Infarction Undergoing Primary Percutaneous Coronary Intervention. KOREAN CIRC J 48: 59-70, 2018 34. Sag S, Yildiz A and Aydin KA, et al.: Association of monocyte to HDL cholesterol level with contrast induced nephropathy in STEMI patients treated with primary PCI. CLIN CHEM LAB MED 55: $132-138,2017$

35. Satilmis S and Karabulut A: Value of C-Reactive Protein/Albumin Ratio in Predicting the Development of Contrast-Induced Nephropathy in Patients With Non-ST Elevation Myocardial Infarction. ANGIOLOGY 71: 366-371, 2020

36. Sun XP, Li J and Zhu WW, et al.: Platelet to Lymphocyte Ratio Predicts Contrast-Induced Nephropathy in Patients With ST-Segment Elevation Myocardial Infarction Undergoing Primary Percutaneous Coronary Intervention. ANGIOLOGY 69: 71-78, 2018

37. Yavuz Karabag MCIR: The C-Reactive Protein to Albumin Ratio Predicts Acute Kidney Injury in Patients With ST-segment Elevation Myocardial Infarction Undergoing Primary Percutaneous Coronary Intervention, 2018

38. Yildirim E, Ermis E and Cengiz M: Inflammatory markers of contrast-induced nephropathy in patients with acute coronary syndrome. CORONARY ARTERY DIS 31: 279-283, 2020

39. Yuan Y, Qiu H and $\mathrm{Hu} X$, et al.: Predictive value of inflammatory factors on contrast-induced acute kidney injury in patients who underwent an emergency percutaneous coronary intervention. CLIN CARDIOL 40: 719-725, 2017

40. Zbierska-Rubinkiewicz K, Trębacz O, Tomala M, Rubinkiewicz M, Chrzan I and Gackowski A: Creatine kinase-MB and red cell distribution width as predictors of contrast-induced nephropathy after percutaneous coronary intervention in acute myocardial infarction. Folia medica Cracoviensia 57: 87, 2017

41. Zhao K, Li Y and Gao Q: Role of red blood cell distribution width in predicting contrast induced nephropathy in patients with stable angina pectoris undergoing percutaneous coronary intervention. INT J CARDIOL 197: 276-278, 2015

42. Zorlu C and Koseoglu C: Comparison of the Relationship Between Inflammatory Markers and Contrast-Induced Nephropathy in Patients With Acute Coronary Syndrome After Coronary Angiography. ANGIOLOGY 71: 249-255, 2020

43. Evola S, Lunetta M and Macaione F, et al.: Risk factors for contrast induced nephropathy: a study among Italian patients. Indian Heart J 64: 484-491, 2012

44. Elhosseiny S, Akel T, Mroue J, Tathineni P, El Sayegh S and Lafferty J: The Value of Adding Red Cell Distribution Width to Mehran Risk Score to Predict Contrast-induced Acute Kidney Injury in Patients with Acute Coronary Syndrome. Cureus 10: e2911, 2018

45. Ridker PM: Clinical application of C-reactive protein for cardiovascular disease detection and 
prevention. CIRCULATION 107: 363-369, 2003

46. Tonelli M, Sacks F, Pfeffer M, Jhangri GS and Curhan G: Biomarkers of inflammation and progression of chronic kidney disease. KIDNEY INT 68: 237-245, 2005

47. Jian-Wei Z, Yu-Jie Z, Shu-Jun C, Qing Y, Shi-Wei Y and Bin N: Impact of preprocedural highsensitivity C-reactive protein on contrast-induced nephropathy in patients undergoing primary percutaneous coronary intervention. ANGIOLOGY 65: 402-407, 2014

48. Yun KH, Lim JH and Hwang KB, et al.: Effect of High Dose Rosuvastatin Loading before Percutaneous Coronary Intervention on Contrast-Induced Nephropathy. KOREAN CIRC J 44: 301-306, 2014

49. Guo XS, Lin KY and Li HL, et al.: Preprocedural High-Sensitivity C-Reactive Protein Predicts Contrast-Induced Nephropathy and Long-Term Outcome After Coronary Angiography. ANGIOLOGY 68: 614-620, 2017

50. Duan C, Cao Y and Liu Y, et al.: A New Preprocedure Risk Score for Predicting Contrast-Induced Acute Kidney Injury. CAN J CARDIOL 33: 714-723, 2017

51. Liu YH, Liu Y and Tan N, et al.: Predictive value of GRACE risk scores for contrast-induced acute kidney injury in patients with ST-segment elevation myocardial infarction before undergoing primary percutaneous coronary intervention. INT UROL NEPHROL 46: 417-426, 2014

52. Lazaros G, Zografos T and Oikonomou E, et al.: Usefulness of C-Reactive Protein as a Predictor of Contrast-Induced Nephropathy After Percutaneous Coronary Interventions in Patients With Acute Myocardial Infarction and Presentation of a New Risk Score (Athens CIN Score). AM J CARDIOL 118: 1329-1333, 2016

53. Toso A, Leoncini M and Maioli M, et al.: Relationship between inflammation and benefits of early high-dose rosuvastatin on contrast-induced nephropathy in patients with acute coronary syndrome: the pathophysiological link in the PRATO-ACS study (Protective Effect of Rosuvastatin and Antiplatelet Therapy on Contrast-Induced Nephropathy and Myocardial Damage in Patients With Acute Coronary Syndrome Undergoing Coronary Intervention). JACC Cardiovasc Interv 7: 1421-1429, 2014

54. Han SS, Kim DK, Kim S, Chin HJ, Chae DW and Na KY: C-Reactive Protein Predicts Acute Kidney Injury and Death After Coronary Artery Bypass Grafting. ANN THORAC SURG 104: 804-810, 2017

55. Fu EL, Franko MA and Obergfell A, et al.: High-sensitivity C-reactive protein and the risk of chronic kidney disease progression or acute kidney injury in post-myocardial infarction patients. AM HEART J 216: 20-29, 2019

56. Tang Y, Huang XR and Lv J, et al.: C-reactive protein promotes acute kidney injury by impairing G1/S-dependent tubular epithelium cell regeneration. Clin Sci (Lond) 126: 645-659, 2014

57. Lai W, Tang Y and Huang XR, et al.: C-reactive protein promotes acute kidney injury via Smad3dependent inhibition of CDK2/cyclin E. KIDNEY INT 90: 610-626, 2016

58. Bian A, Shi M and Flores B, et al.: Downregulation of autophagy is associated with severe ischemiareperfusion-induced acute kidney injury in overexpressing C-reactive protein mice. PLOS ONE 12: e181848, 2017

59. Zahler D, Rozenfeld K and Stein M, et al.: C-reactive protein velocity and the risk of acute kidney injury among ST elevation myocardial infarction patients undergoing primary percutaneous intervention. J NEPHROL 32: 437-443, 2019

60. Gaetano M. De Ferrari SCAS and Marco Ferlini SPMF: platelet to lymphocyte Ratio is an independent Risk Factor of contrast induced nephropathy in patients with st elevation myocardial infarction undergoing 
primary percutaneous coronary intervention. Poster Contributions

Poster Hall B1

Monday, March 16, 2015, 9:45 a.m.-10:30 a.m, 2015

61. Velibey Y, Oz A and Tanik O, et al.: Platelet-to-Lymphocyte Ratio Predicts Contrast-Induced Acute Kidney Injury in Patients With ST-Segment Elevation Myocardial Infarction Undergoing Primary Percutaneous Coronary Intervention. ANGIOLOGY 68: 419-427, 2017

62. Turkmen K, Erdur FM and Ozcicek F, et al.: Platelet-to-lymphocyte ratio better predicts inflammation than neutrophil-to-lymphocyte ratio in end-stage renal disease patients. HEMODIAL INT 17: 391-396, 2013

63. Jiang J, Ji H and Xie W, et al.: Could platelet-to-lymphocyte ratio be a predictor for contrast-induced nephropathy in patients with acute coronary syndrome? MEDICINE 98: e16801, 2019

64. Shah N, Parikh V and Patel N, et al.: Neutrophil lymphocyte ratio significantly improves the Framingham risk score in prediction of coronary heart disease mortality: insights from the National Health and Nutrition Examination Survey-III. INT J CARDIOL 171: 390-397, 2014

65. Kurtul A, Yarlioglues M, Duran M and Murat SN: Association of Neutrophil-to-lymphocyte Ratio with Contrast-induced Nephropathy in Patients with Non-ST-elevation Acute Coronary Syndrome Treated with Percutaneous Coronary Intervention. HEART LUNG CIRC 25: 683-690, 2016

66. Vuruskan E and Saracoglu E: Bilirubin Levels are Associated With Contrast-Induced Nephropathy in Peripheral Artery Disease. ANGIOLOGY 68: 728-733, 2017

67. Argan O, Ural D and Kozdag G, et al.: Associations Between Neutrophil Gelatinase Associated Lipocalin, Neutrophil-to-Lymphocyte Ratio, Atrial Fibrillation and Renal Dysfunction in Chronic Heart Failure. Med Sci Monit 22: 4765-4772, 2016

68. Azab B, Daoud J and Naeem FB, et al.: Neutrophil-to-lymphocyte ratio as a predictor of worsening renal function in diabetic patients (3-year follow-up study). Ren Fail 34: 571-576, 2012

69. Mehran R, Aymong ED and Nikolsky E, et al.: A simple risk score for prediction of contrast-induced nephropathy after percutaneous coronary intervention: development and initial validation. J AM COLL CARDIOL 44: 1393-1399, 2004

70. Nikolsky E, Mehran R and Lasic Z, et al.: Low hematocrit predicts contrast-induced nephropathy after percutaneous coronary interventions. KIDNEY INT 67: 706-713, 2005

71. Tonelli M, Sacks F, Arnold M, Moye L, Davis B and Pfeffer M: Relation Between Red Blood Cell Distribution Width and Cardiovascular Event Rate in People With Coronary Disease. CIRCULATION 117: 163-168, 2008

72. Akin F, Kose N and Ayca B, et al.: Relation between red cell distribution width and severity of coronary artery disease in patients with acute myocardial infarction. ANGIOLOGY 64: 592-596, 2013

73. Docci D, Delvecchio C, Gollini C, Turci F, Baldrati L and Gilli P: Red blood cell volume distribution width (RDW) in uraemic patients on chronic haemodialysis. INT J ARTIF ORGANS 12: 170-174, 1989

74. Ujszaszi A, Molnar MZ, Czira ME, Novak M and Mucsi I: Renal function is independently associated with red cell distribution width in kidney transplant recipients: a potential new auxiliary parameter for the clinical evaluation of patients with chronic kidney disease. Br J Haematol 161: 715 725,2013

75. Van Driest SL, Jooste EH and Shi Y, et al.: Association Between Early Postoperative Acetaminophen Exposure and Acute Kidney Injury in Pediatric Patients Undergoing Cardiac Surgery.

JAMA PEDIATR 172: 655-663, 2018 
76. Zou Z, Zhuang Y and Liu L, et al.: Role of elevated red cell distribution width on acute kidney injury patients after cardiac surgery. BMC CARDIOVASC DISOR 18: 166-167, 2018 